\title{
On Advertising Games and Spillover in Service Systems
}

\author{
Lei Xu, ${ }^{1,2}$ Wang Zhigang, ${ }^{3}$ Jun Shan, ${ }^{2}$ and Yanzhi Wang ${ }^{4}$ \\ ${ }^{1}$ School of Management, Tianjin University of Technology, Tianjin 300384, China \\ ${ }^{2}$ Business School, Nankai University, Tianjin 300071, China \\ ${ }^{3}$ School of Electrical Engineering, Tianjin University of Technology, Tianjin 300384, China \\ ${ }^{4}$ Business School, Tianjin University of Commerce, Tianjin 300134, China
}

Correspondence should be addressed to Jun Shan; jshan@nankai.edu.cn

Received 22 July 2013; Revised 18 September 2013; Accepted 6 October 2013

Academic Editor: Pui-Sze Chow

Copyright (C) 2013 Lei Xu et al. This is an open access article distributed under the Creative Commons Attribution License, which permits unrestricted use, distribution, and reproduction in any medium, provided the original work is properly cited.

\begin{abstract}
Motivated by the industry cases, we model the advertising competition between the dominant service provider and small service providers in one service market, where the dominant service provider has a major market share and the other small service providers share the remainder of market equally. Based on this setting, we discuss three advertising game models, that is, cooperative game, Boxed Pig game, and Prisoner's game, derive the conditions for different advertising games, and characterize their equilibria. To be specific, it is found that the advertising spillover and the number of the small service providers directly determines the advertising game equilibria, while other market parameters, to some extent, can affect the results of the advertising game equilibria. According to our theoretical findings, some management insights and suggestions are given from both the academic and practical perspectives.
\end{abstract}

\section{Introduction}

When you watch TV, listen to radio, read newspapers and magazines, or just surf on the internet, various kinds of exquisite advertisements immediately appear into your eyes. They compete to deliver a claim to customers that "buy me, you deserve!" Undoubtedly, advertising plays an important role in the product sales [1], as well as in enhancing customers' stickiness to the objective brand [2]. In the homogenization of service market, in order to be differentiated from other competitors, many service providers spend heavily on advertising; hoping to obtain special favor of consumers through constructing brand of company, advertising has been becoming a major strategy of nonprice competitions [3]. In general, service providers use two kinds of advertising to promote sales and to strengthen their brands, namely, generic advertising and brand advertising. The effect of generic advertising is to increase category sales while not mentioning the sponsoring brand. In contrast, brand advertising aims to gain market share by providing information about brand's value to consumers $[4,5]$.

Service providers adopt different advertising strategies in different market structures: a monopolistic service provider chooses the optimal advertising from its own perspective; in the oligopolistic market, however, service providers cannot achieve the expected objectives if they only consider their own advertising decisions. In order to ensure market shares and strengthen market positions, service providers, in this oligopolistic market, must take into account other service providers' advertising strategies. For example, China Life Insurance Co., Ltd. is a famous insurance company, which provides a variety of insurance products to customers and once occupied most of the shares of insurance market. However, with the powerful competitors such as Ping An Insurance company of China, Ltd. and Tai Kang Life Insurance Co., Ltd. entering into the insurance market, China Life Insurance Co., Ltd. has to adjust the marketing strategy and pay more attention on strengthening brand image in advertising, which aims to consolidate the market share in the fierce competition.

Furthermore, service providers' advertising strategies are also influenced by their upstream suppliers. In order to obtain the spillover effects of advertisings, the upstream suppliers always induce downstream service providers to invest more on advertising. For instance, the suppliers can offer subsidies to incentivize the downstream service providers to advertise 
more, in order to improve the overall channel profits and competitive advantages.

In this paper, we try to answer the following research questions about the advertising investment issues under a competitive market environment. How should service providers decide the optimal advertising investment in a competitive environment? If one service provider acts a dominate player in the market, should it invest in advertising first or wait the small service providers to advertise? As for a small service provider, what strategy should it adopt, advertising or waiting? And what is the most efficient strategy for the whole market?

The remainder of this paper is organized as follows. In Section 2, we review the related literature. Section 3 describes the problem and presents the advertising game models. The analysis of the game models is given in Section 4. Finally, we conclude the paper in Section 5.

\section{Literature Review}

There are mostly three areas of research relevant to our work: first, duopolistic advertising, second competitive game models on advertising, and third cooperative game models of advertising.

A stream of the literature has used game theoretical models to study the advertising investment issue in a duopoly setting. Espinosa \& Mariel [6] find that, for the informative advertising competition, the outcomes from advertising are closer to the collusive outcomes in a feedback equilibrium. But in the case of predatory advertising, they find that expenditures are inefficiently high in a feedback equilibrium and the open-loop solution is more efficient. Prasad \& Sethi [7] analyze the optimal advertising spending in a duopolistic market, develop a differential advertising game, in which the retailers' dynamic behavior is based on the Sethi stochastic advertising model and Lanchester combat model, and derive the closed-loop Nash equilibrium for symmetric and asymmetric competitors. Consistent with previous studies, Bass et al. [8] suggest that in a dynamic duopoly, generic advertising is more important than brand advertising for the long-run market shares, but in the short-term period, brand advertising is effective and market potential saturation leads to a decline in generic advertising over time.

However, little attention has been paid to advertising competition in an oligopolistic market setting, which is more common in practice. In addition, most of existing studies are based on the differential game theory, which only covers the dynamics features in advertising competition [9]. More research methods on advertising competition should be adopted to investigate other features of advertising competition, in particular, other game theories in the oligopoly market. Some recent literature has put effort to fill this gap. Brady [10] uses the Cournot game model to discuss the retailers' optimal advertising decisions, and the simulation analysis shows that small changes in model parameters can significantly influence the retailers' advertising behavior. Viscolani [11] suggests that two retailers, producing substituting products in the homogenization market, advertise to influence the product demand and interfere negatively with each other. He derives the pure-strategy Nash equilibrium and analyzes its characteristics in an advertising game. Nevertheless, these studies still concentrate on the duopoly market setting. Considering an oligopolistic market with multiple retailers who sell and advertise for a homogenous product, Norman et al. [12] find that the advertising intensity is related to the market concentration but make no discussion on the issue how retailers of different size make their optimal advertising decisions in the oligopolistic market.

Another stream of the related literature is on the cooperative advertising in the vertical supply chains. For instance, He et al. [13] use a differential Stackelberg game model to investigate the cooperative advertising between the manufacturers and retailers in the supply chain. He compares the advertising and pricing decisions in between the vertical integrated channel and coordinated channel. Seyedesfahani et al. [14] discuss three vertical noncooperative advertising games, including Nash, Stackelberg, and a cooperative game. They find that in the cooperative game, manufacturers and retailers can obtain the largest profits. However, none of the existing literature considers how the cooperative advertising in the supply chain level affects the advertising competition in the downstream market. Our paper contributes to this literature by considering the cooperation between the service providers. Moreover, few studies have investigated repeated competition game in the advertising competition.

In this paper, we construct the advertising models between the dominant service providers and small service providers in a market, discuss three advertising game models, that is, cooperative game, Boxed Pig game and Prisoner's game (in Boxed Pig game, a big, dominant pig and a little, weaker pig are locked into a Skinner box. At one end is a food dispenser. Ten units of food/energy are dispensed when a panel at the other end of the box is pressed. The pigs must each decide whether to wait by the food dispenser or to expend 2 units of energy to press the panel and run to the food. The big pig can run faster and eat faster than the little pig, so the little pig knows it will do better if it gets to the dispenser before the big pig. The pigs' actions are simultaneous, so the pigs must predict each other's strategy and then decide on their own best strategy. For more details, please refer to Rasmussen (1989) and in a conventional version of the game, two prisoners are interrogated separately about a bank robbery. If one confesses to the robbery and the other does not, the one who confesses is released, and the other receives a stiff sentence. If both confess, they receive moderate sentences. If neither confesses, they receive mild sentences for a lesser crime. Each player follows a dominant strategy, which is a strategy that is the player's best response, the one with the highest payoff, not matter what strategy the other player chooses. Assuming at atmosphere of distrust and competition, the dominant strategy equilibrium of the game is for both prisoners to confess, even though the Pareto superior outcome, the one in which neither party can be made better off, is, for both to keep quiet. For more details, please refer to Mudambi (1996)) and derive the conditions for different advertising games and their equilibrium, respectively. The main contributions of this paper are summarized as follows. First of all, the 
paper considers the advertising competitions among service providers and divides them into two types according to the service providers' size. Besides, this paper also considers the advertising subsidies from upstream service providers to downstream service providers affecting the competitions in the oligopolistic market.

\section{Model Developments}

3.1. Problem Descriptions and Notations. We consider a competitive market consisting of $M+1$ risk-neutral service providers, among which a dominant service provider (denoted by $D$ for short) has a majority market share $w_{D}$, and the other $M$ small service providers share the remaining market share $1-w_{D}$ equally. Each service provider's market share is denoted by $w_{i}(i=1,2, \ldots, M)$, and $w_{D} \gg w_{i}$, $w_{D}+\sum_{i=1}^{M} w_{i}=1$. The dominant service provider $D$ and small service providers play advertising investment games. We take the large service provider $D$ and one classical small service provider $S$ as examples to analyze the service providers' advertising strategies.

We model the problem as a Nash game between service providers $D$ and $S$. Service provider $D$ (or $S$ ) can decide to advertise with probability $p$ (or $q$ ) or to wait with probability $1-p($ or $1-q)$. And it is assumed that the other $M-1$ small service providers act in the same way where they do not invest in advertising and wait service providers $D$ or $S$ to provide advertising. Here, the wait strategy means that the service provider does not invest in advertising and wait other service providers to provide advertising. The other $M-1$ small service providers can share the advertising effects of service provider $D$ and $S$, which is consistent with real cases, and this is so-called "spillover effect." (Spillover effect refers to the extent to which information provided in messages changes beliefs about attributes that are not mentioned in the messages [15].) We assume that $\theta$ is advertising spillover rate, and $\theta=\alpha M(0<\theta<1)$.

Based on the service providers $D$ and $S$ ' different choice of advertising strategies, cooperative advertising and waiting, the service providers' decision pairs can be divided into four cases.

Case 1 (cooperative advertising). The total investment on advertising is $I$ (including labor, material, and technique), where $a I$ is for the service provider $D$ and $b I$ for the service provider $S$, where $a+b=1$. We assume service provider $D$ has more advertising resources and will invest more on advertisement. To capture this feature, we denote $l(l>1)$ to be the resource coefficient such that $a I=l b I$. Letting $k$ denote the effect of cooperative advertising, where $k>1$, the revenue stemmings from the cooperative advertising are $R_{D 1}=\mathrm{kblI}$ and $R_{S 1}=k b I$.

Case 2 (service provider $D$ advertises, but service provider $S$ waits). Service provider D's advertising investment is $a I$, the advertising effects coefficient is $k_{D}$, the total revenue from service provider D's advertising is $R_{D 2}=b l k_{D} I$.
TABLE 1: Advertising Game Matrix between service providers $D$ and $S$.

\begin{tabular}{cccc}
\hline \multicolumn{3}{c}{ Provider $S$} \\
Provider $D$ & $\begin{array}{c}\text { Strategy } \\
\text { (probability) }\end{array}$ & Advertising $(q)$ & Waiting $(1-q)$ \\
& Advertising $(p)$ & $\pi_{D 1}, \pi_{S 1}$ & $\pi_{D 2}, \pi_{S 2}$ \\
& Waiting $(1-p)$ & $\pi_{D 3}, \pi_{S 3}$ & $\pi_{D 4}, \pi_{S 4}$ \\
\hline
\end{tabular}

Case 3 (service provider $S$ advertises, but service provider $D$ waits). Retail $S$ 's advertising investment is $b I$, advertising effects coefficient is $k_{S}$, the total revenue is $R_{S 3}=b k_{S} I$, and $k>k_{D}>k_{S}$.

Case 4 (noncooperative advertising (Prisoner's dilemma)). Both service provider $D$ and service provider $S$ choose to wait, and the payoff is $R_{i 4}=0, i=D, S$.

Because of the advertising spillover problem, the service provider who offers advertising cannot obtain the whole advertising effect. Therefore, in order to cultivate the service providers to advertise, the supplier offers a subsidy rate $\lambda$ to the service provider who invests on advertising for his products, so that the total subsidy amount is $\lambda R$. (In this paper, we do not consider the supplier's decisions and his revenue, we mainly study the impact of the subsidy on both service providers' decisions. For the subsidy on the revenue, in fact, it is related to the advertising investment $I$ indirectly, because the revenue is a function of advertising investment. It is noted that this setting can solve the subsidy difference between the service providers $D$ and $S$ using one subsidy rate.) Suppose the subsidy rate $\lambda$ is the same for different advertising effects. Throughout the paper, we use he and she to indicate service providers $D$ and $S$, respectively.

3.2. Advertising Game Models. In the previously mentioned settings, we can construct the payoff matrix of service providers $D$ and $S$ as shown in Table 1. Obviously, the other small service providers act in the same way as service provider $S$.

Based on the previously mentioned analysis, we obtain the service providers $D$ and $S$ ' payoff functions as follows.

Case 1. Both service providers $D$ and $S$ choose to invest in advertising.

Both service providers $D$ and $S$ ' payoff functions are given as follows:

$$
\begin{gathered}
\pi_{D 1}=(1-\theta) k b l I-b l I+\lambda k b l I, \\
\pi_{S 1}=(1-\theta) k b I-b I+\lambda k b I,
\end{gathered}
$$

where the first term in (1) is service provider D's revenue from the advertising strategy, the second term is the advertising expenses, and the third term is the subsidy from the supplier. Similarly, (2) indicates service provider $S$ 's payoff function. 
With the same logic, in Case 2 , service providers $D$ and $S$ ' payoff functions are given as follows:

$$
\begin{gathered}
\pi_{D 2}=(1-\theta) k_{D} b l I-b l I+\lambda k_{D} b l I, \\
\pi_{S 2}=\frac{\theta}{M} k_{D} b l I,
\end{gathered}
$$

where the first term in (3) is service provider D's revenue from the advertising strategy, the second term is the advertising expenses, and the third term is the subsidy from the supplier. In (4), since service provider $S$ chooses to wait, instead of offering advertisement, there is no cost incurred on advertising.

In Case 3, service providers $D$ and $S^{\prime}$ payoff functions are given as follows:

$$
\begin{gathered}
\pi_{D 3}=\frac{\theta}{M} \beta k_{S} b l I, \\
\pi_{S 3}=(1-\theta) k_{S} b I-b I+\lambda k_{S} b I,
\end{gathered}
$$

where the term $(\theta / M) \beta$ indicates the advertising spillover rate that service provider $D$ obtains from service provider $S$ 's advertising and $\beta(\beta>1)$ is the service provider $D$ 's spillover factor. In this case, service provider $D$ chooses to wait and the small service provider $S$ chooses to offer advertisement.

In Case 4, no service provider chooses to offer advertisement; thus the service providers' payoff functions are

$$
\pi_{D 4}=\pi_{S 4}=0 .
$$

Since both service providers choose to wait, there is no revenue generated. This leads to a well-known game resultPrisoner's dilemma.

\section{Game Model Analysis}

In this section, we will analyze the equilibrium of cooperative game and Boxed Pig game and then further study the deterioration of the Boxed Pig game.

4.1. Cooperative Equilibrium. In this advertising game, service provider $D$ has complete information on his own and incomplete information on service provider $S$. Service provider D's payoff difference between advertising and waiting strategy is $\Delta \pi_{D}$, and whether he chooses to advertise or not lies on his expected payoff differences.

According to the analysis in Section 4.1, his payoff difference is

$$
\Delta \pi_{D}=\sum_{i=1}^{4} p_{i} q_{i} \pi_{D i}(p=1)-\sum_{i=1}^{4} p_{i} q_{i} \pi_{D i}(p=0) \geq 0
$$

where $\pi_{D i}(p=1)$ is service provider D's payoff when he choose to invest in advertising, and $\pi_{D i}(p=0)$ represents service provider $D$ 's payoff when he choose to wait.

If and only if $\Delta \pi_{D} \geq 0$ holds, service provider $D$ chooses the cooperative advertising strategy.

Where $i=1,2,3$, and $4, p_{1}=p_{2}=p, p_{3}=p_{4}=1-p$, $q_{1}=q_{3}=q$, and $q_{2}=q_{4}=1-q$.
Substituting the previously mentioned conditions into $\Delta \pi_{D}$, we obtain

$$
\begin{aligned}
\Delta \pi_{D}=\{ & q[(1-\theta) k+\lambda k]+(1-q) \\
& \left.\times\left[(1-\theta) k_{D}-1+\lambda k_{D}\right]-q \frac{\theta}{M} \frac{k_{S} \beta}{l}\right\} b l I .
\end{aligned}
$$

Since $\theta=\alpha M$ and $\Delta \pi_{D} \geq 0$, the sufficient and necessary conditions for service provider $D$ to choose to advertise are

$$
\begin{gathered}
\theta_{D} \leq 1-\frac{q \alpha\left(k_{S} \beta / l\right)-\lambda k q-\lambda k_{D}(1-q)+1}{k q(1+\delta)^{n-1}+k_{D}(1-q)}=\bar{\theta}_{D}, \\
M_{D} \leq \frac{1}{\alpha}-\frac{q \alpha\left(k_{2} \beta / l\right)-\lambda k q-\lambda k_{D}(1-q)+1}{\alpha k q+\alpha k_{D}(1-q)}=\bar{M}_{D},
\end{gathered}
$$

respectively.

Service provider $S$ 's payoff difference between advertising and waiting strategy is $\Delta \pi_{S}$. For service provider $S$, the sufficient and necessary condition for her to choose the cooperative advertising strategy is

$$
\Delta \pi_{S}=\sum_{i=1}^{4} p_{i} q_{i} \pi_{S i}(q=1)-\sum_{i=1}^{4} p_{i} q_{i} \pi_{S i}(q=0) \geq 0 .
$$

Substituting the previously mentioned state probability condition into $\Delta \pi_{S}$, we obtain

$$
\begin{aligned}
\Delta \pi_{S}=\{p & {[(1-\theta) k+\lambda k]+(1-p) } \\
& \left.\times\left[(1-\theta) k_{S}-1+\lambda k_{S}\right]-q \frac{\theta}{M} k_{D} l\right\} b I \geq 0 .
\end{aligned}
$$

In a similar way, since $\theta=\alpha M$ and $\Delta \pi_{S} \geq 0$, we can derive that the sufficient and necessary conditions for service provider $S$ to choose the cooperative advertising strategy are

$$
\begin{gathered}
\theta_{S} \leq 1-\frac{p \alpha k_{D} l-\lambda k q-\lambda k_{S}(1-p)+1}{k p+k_{S}(1-p)}=\bar{\theta}_{S}, \\
M_{S} \leq \frac{1}{\alpha}-\frac{p \alpha k_{D} l-\lambda k q-\lambda k_{S}(1-p)+1}{\alpha k p+\alpha k_{S}(1-p)}=\bar{M}_{S} .
\end{gathered}
$$

With the previous analytical results, we can derive the following theorem.

Theorem 1. The sufficient and necessary conditions for service providers $D$ and $S$ to choose the cooperative advertising strategy are that there exists a spillover rate upper bound, that is, $\bar{\theta}_{i}$ and $i=D, S$, meanwhile, an upper bound for the number of small service provider, that is, $\bar{M}_{i}$ and $i=D, S$, under which both players choose to advertise simultaneously. Otherwise, both service providers $D$ and $S$ choose to wait.

Considering that both service providers $D$ and $S$ have the same cooperation preferences; that is, the probabilities of cooperative advertising strategy are the same for service 
providers $D$ and $S(p=q)$ and given the advertising spillover rate $\theta$, the subsidy $\lambda$, and the advertising effect of service provider SblI, we can derive the following conditions for service provider $D$ and $S$ to cooperate on advertising:

$$
\overline{\theta_{S}} \leq \overline{\theta_{D}}, \quad \overline{M_{S}} \leq \overline{M_{D}} .
$$

Under the previously mentioned conditions, the binding constraint conditions for service provider $D$ and $S$ are

$$
\begin{aligned}
& \theta_{e} \leq \overline{\theta_{S}}=1-\frac{p \alpha k_{D} l-\lambda k q-\lambda k_{S}(1-p)+1}{k p+k_{S}(1-p)}, \\
& M_{e} \leq \overline{M_{S}}=-\frac{p \alpha k_{D} l-\lambda k q-\lambda k_{S}(1-p)+1}{\alpha k p+\alpha k_{S}(1-p)} .
\end{aligned}
$$

The equilibrium of both service providers choosing the cooperative advertising strategies is the most efficient state. As long as the condition $(1-\theta) k \geq 1$ holds, $\Delta \pi=\pi_{D 1}+\pi_{S 1}$ $\left(\pi_{D 2}+\pi_{S 2}\right) \geq 0$. This result indicates that the efficiency of the cooperative advertising strategy equilibrium is no less than that in Boxed Pig game.

The previously mentioned analysis also indicates that when the advertising spillover rate $\theta$ is not very large, the subsidy parameter $\lambda$ and the discount rate $\delta$ are larger; the cooperative advertising strategy will be the equilibrium outcome.

\subsection{Equilibrium of Boxed Pig Game and Deterioration. Boxed} Pig game cannot attain the Pareto optimality, but the equilibrium is still more efficient compared to that of Prisoner's dilemma. In the following, we aim to analyze the equilibrium conditions of Boxed Pig game and how it deteriorates into Prisoner's dilemma game.

Suppose that both service providers $D$ and $S$ have the same willingness of cooperative advertising strategy, that is, $p=q$; thus in certain condition of the spillover rate $\theta$, the subsidy parameter $\lambda$ and the advertising effect $b l I$ of service provider $S$, we can derive the following conditions in which only one service provider chooses to offer advertisement, but the other service providers will choose to wait and reap the payoff:

$$
\overline{\theta_{S}} \leq \theta_{e} \leq \overline{\theta_{D}} \quad \text { or } \quad \overline{M_{S}} \leq M_{e} \leq \overline{M_{D}} .
$$

More specifically, the previously mentioned conditions can be rewritten as

$$
\begin{aligned}
1- & \frac{p \alpha k_{D} l-\lambda k p-\lambda k_{S}(1-p)+1}{k p+k_{S}(1-p)} \\
& \leq \theta_{e} \\
& \leq 1-\frac{q \alpha\left(k_{S} \beta / l\right)-\lambda k q-\lambda k_{D}(1-q)+1}{k q+k_{D}(1-q)},
\end{aligned}
$$

or

$$
\begin{aligned}
\frac{1}{\alpha} & -\frac{p \alpha k_{D} l-\lambda k p-\lambda k_{S}(1-p)+1}{\alpha k p+\alpha k_{S}(1-p)} \\
& \leq M_{e} \\
& \leq \frac{1}{\alpha}-\frac{q \alpha\left(k_{S} \beta / l\right)-\lambda k q-\lambda k_{D}(1-q)+1}{\alpha k q+\alpha k_{D}(1-q)} .
\end{aligned}
$$

By the previously mentioned analytical results, we can easily obtain the conditions in which Boxed Pig game deteriorates into Prisoner's dilemma equilibrium, that is, (waiting, waiting):

$$
\theta_{e}>\overline{\theta_{D}} \quad \text { or } \quad M_{e}>\overline{M_{D}} \text {. }
$$

In the previous conditions, either service provider chooses to wait the other service provider to offer advertisement; however, neither service provider chooses to offer advertisement eventually.

4.3. Analyses on Equilibrium of Cooperative Game and Boxed Pig Game. The advertising strategy equilibrium depends on the values of $\theta_{e}, \overline{\theta_{D}}, \overline{\theta_{S}}, M_{e}, \overline{M_{D}}$, and $\overline{M_{S}}$. Next, we will employ the comparative static analysis to discuss factors relevant to $\theta$ and $M$, such as the subsidy $\lambda$, the advertising effects parameters $k, k_{D}$, and $k_{S}$, the resource power $l$, and the discount factor $\delta$.

With respect to (9) and (12), taking first-order derivatives of $\overline{\theta_{D}}, \overline{\theta_{S}}, \overline{M_{D}}$, and $\overline{M_{S}}$ on $\lambda$, respectively, we have,

$$
\begin{aligned}
\frac{\partial \overline{\theta_{D}}}{\partial \lambda}>0, & \frac{\partial \overline{\theta_{S}}}{\partial \lambda}>0, \\
\frac{\partial \overline{M_{D}}}{\partial \lambda}>0, & \frac{\partial \overline{M_{S}}}{\partial \lambda}>0 .
\end{aligned}
$$

The previous results show that first-order derivatives on the subsidy $\lambda$ are bigger than zero, which implies that the spillover rate both service providers can tolerate and the number of small service providers are increasing with respect to the subsidy $\lambda$ from the supplier. In other words, with the subsidy increases, both service providers $D$ and $S$ have larger spillover rates and prefer to offer advertisement. Then, we obtain Proposition 2 as follows.

Proposition 2. The more the subsidy ( $\lambda$ ) from the supplier is, the more both service providers prefer to choose to offer advertisement.

This proposition has an implication that the subsidy from the supplier makes both service providers able to tolerate a larger spillover rate $\theta$ and the number of the small service providers, because both service providers can compensate this loss stolen by the small service providers with the subsidy. 
With respect to (9) and (12), taking first-order derivatives of $\overline{\theta_{D}}, \overline{\theta_{S}}, \overline{M_{D}}$, and $\overline{M_{S}}$ on $k_{D}$ and $k_{S}$, respectively, we have

$$
\begin{aligned}
\frac{\partial \overline{\theta_{D}}}{\partial k_{D}}>0, & \frac{\partial \overline{\theta_{S}}}{\partial k_{S}}>0, \\
\frac{\partial \overline{M_{D}}}{\partial k_{D}}>0, & \frac{\partial \overline{M_{S}}}{\partial k_{S}}>0 .
\end{aligned}
$$

The previous results show that with the advertising effects increase, service providers $D$ and $S$ prefer to cooperate on advertising more. No fairness preferences are considered, both service providers maximize their payoff. Then, we can obtain Proposition 3 as follows.

Proposition 3. The more the advertising effect $\left(k_{D}, k_{S}\right)$ is, the more both service providers prefer to choose to offer advertisement.

This proposition implies that the advertising effect makes both service providers tolerate a market with a large spillover rate $\theta$ and where a number of the small service providers freeride, because both service providers can obtain more payoff from the advertising strategy.

With respect to (9) and (12), taking first-order derivatives of $\overline{\theta_{D}}, \overline{M_{D}}$ on $k_{S}$, and $\overline{\theta_{S}}, \overline{M_{S}}$ on $k_{D}$, respectively, we obtain the following results:

$$
\begin{array}{ll}
\frac{\partial \overline{\theta_{D}}}{\partial k_{S}}<0, & \frac{\partial \overline{M_{D}}}{\partial k_{S}}<0, \\
\frac{\partial \overline{\theta_{S}}}{\partial k_{D}}<0, & \frac{\partial \overline{M_{S}}}{\partial k_{D}}<0 .
\end{array}
$$

The previous results show that the first-order partial derivatives are less than 0 . This finding means that when service provider $D$ knows that service provider $S$ has a higher advertising effect, service provider $D$ will choose to wait, because service provider $S$ will offer advertisement to maximize his payoff, and vice versa. On the other hand, this easily tends towards a Boxed Pig game, where the service provider with less advertising effect will wait for service providers with more advertising effect to offer advertisement, so that he could share the advertising spillover effects. We obtain the following proposition.

Proposition 4. The more the service provider D's advertisement advertising effect is, the less service provider $S$ is willing to offer advertisement, and vice versa.

The results are in accord with real cases: if one service provider has more advertising resource, the other service provider would prefer to believe that the service provider who has more advertising resource will offer advertisement; as a result, this service provider will choose to wait for the advertising spillover. Theoretically, when his rival has a more advertising effect, the service provider can tolerate a smaller advertising spillover rate $\theta$ and the number of the small service provider $M$, and vice versa.
With respect to (9) and (12), taking first-order derivatives of $\overline{\theta_{D}}, \overline{\theta_{S}}, \overline{M_{D}}$, and $\overline{M_{S}}$ on $l$, we obtain that

$$
\begin{aligned}
\frac{\partial \overline{\theta_{D}}}{\partial l}>0, & \frac{\partial \overline{M_{D}}}{\partial l}>0, \\
\frac{\partial \overline{\theta_{S}}}{\partial l}<0, & \frac{\partial \overline{M_{S}}}{\partial l}<0 .
\end{aligned}
$$

The previous conditions indicate that given service provider $S$ 's advertising investment, the more the service provider $D$ invests, the less the number of the service providers and the spillover rate that service provider $S$ can tolerate.

Proposition 5. The more the service provider D's advertising resource is, the larger the advertising spillover rate he can tolerate. Meanwhile, the less the advertising spillover rate and the number of the small service provider that service provider $S$ can tolerate.

This proposition shows that when the service provider $D$ 's advertising resource affects service provider $D$ and $S$ ' tolerance of the advertising spillover rate and the number of the small service provider in the market simultaneously, and with the advertising resource increases, service provider $D$ can tolerate a market with a larger advertising spillover; however, service provider $S$ can obtain fewer payoff; then she will choose to wait service provider $D$ to offer advertisement.

With respect to (9) and (12), taking the first-order derivative of $\overline{\theta_{D}}$ and $\overline{M_{D}}$ on $\beta$, we obtain

$$
\frac{\partial \overline{\theta_{D}}}{\partial \beta}<0, \quad \frac{\partial \overline{M_{D}}}{\partial \beta}<0 .
$$

This result shows that given his advertising resource, in order to obtain more revenue from the advertising, service provider $D$ has to reduce the influencing factor of advertising spillover and lessen small service providers he can tolerate in the market.

Proposition 6. At equilibrium (waiting, advertising), the larger the spillover rate of service provider D can obtain from the advertising is, the smaller the advertising spillover rate and the number of the small service providers service provider $D$ can tolerate.

Let $k_{1}=\gamma k_{2}$ and $\gamma>1$, where $\gamma$ denotes the comparison of advertising resource between service providers $D$ and $S$. A larger comparison ratio means a larger advertising resource difference between service providers $D$ and $S$.

Combing $k_{1}=\gamma k_{2}$ into (9) and (12) and taking the firstorder derivatives of $\overline{\theta_{D}}$ and $\overline{\theta_{S}}$ on $\gamma$, we can obtain that

$$
\frac{\partial \overline{\theta_{D}}}{\partial \gamma}>0, \quad \frac{\partial \overline{\theta_{S}}}{\partial \gamma}<0 .
$$

From the previous conditions, we can derive the following proposition. 
Proposition 7. The larger the advertising resource comparison ratio $\gamma$ is, the larger the advertising spillover rate $\theta$ service provider $D$ can tolerate and service provider $D$ chooses to cooperate on advertising, and vice versa.

This proposition indicates that if the advertising can bring more revenues to service providers in the market, more service providers choose to cooperate on advertising, not only the dominate service provider $D$, but also the follower service providers.

Moreover, when the dominant service provider $D$ can obtain more revenue from the advertising that small service providers, that is, a larger $\gamma$, service provider $D$ will choose to offer advertisement, no matter whether the small service provider responses.

\section{Conclusions and Future Research}

Motivated by the industry case given in the introduction section, we constructed the advertising models between the dominant service provider and small service providers in a market. In our model, the dominant service provider has a major market share, while $M$ small service providers have the remaining market share. Based on this setting, we discussed three advertising game models (i.e., cooperative game, Boxed Pig game, and Prisoner's game) and characterized the conditions for different advertising games and their equilibria, respectively. To be specific, it is found that the advertising spillover and the number of the small service providers directly determines the advertising game equilibria, and other market parameters, to some extent, can also affect the results of the advertising game equilibria as well.

According to our theoretical findings, we make the following suggestions on choices of the advertising strategies for the supplier, the dominate service provider and small service providers. For service providers, first of all, they can realize resource complementarities and achieve the advertising synergy effects through a cooperative advertising. On the contrary, non-cooperative advertising is not a wise alternative, because the spillover effect can improve service provider's short-term profits at the expense of the long-term profits. Therefore, service providers should cooperate with each other in order to achieve long-term and whole supply chain revenue instead of free ride.

For suppliers, first, the larger the number of small service provider $M$ is, the higher the probability for small service providers choosing to wait is. Therefore, it is necessary to reduce the number of service providers in the market in order to promote downstream service providers to cooperate on advertising. Second, suppliers should consider the type of service providers and offer different subsidies to the dominant service provider and small service providers. Finally, suppliers should foster relatively large service providers because large service providers tend to offer advertisement actively, and large service provider can signal advertising strategy to small service providers to cooperate with them.

This analysis can be extended in several directions. In practice, the service quality is the basic condition for the service providers to invest more in advertising; then a research on service quality is an interesting question in future [16]. The supplier and service providers cooperate and compete on advertising in many different forms. A subsidy between the supplier and service providers is only one of them. Thus, a more comprehensive investigation of interaction between the supplier and service providers would be a promising research direction. Furthermore, we only consider an advertising decision on whether to offer an advertisement or not in our current model. However, why and how to offer advertisement are not covered. It would be interesting to further analyze the specific advertising strategies of different service providers.

\section{Acknowledgments}

The authors thank the editor, associate editor, and three anonymous reviewers for their constructive suggestions, which have improved this paper tremendously. The work of Lei $\mathrm{Xu}$ was supported in part by the National Natural Science Foundation of China under Grants 71302005, 71002070, and 71002106, and in part by scientific development strategy Project of Tianjin under Grants 12ZLZLZF07700 and 13ZLZLZF03500. The work of Jun Shan was partially supported by the National Natural Science Foundation of China under Grant 71102048, and partially by the Humanities and Social Science Research Funds of Ministry of Education of China under Grant 10YJC630200, which are gratefully acknowledged by the authors.

\section{References}

[1] K. Olorunleke and S. T. Akinyele, "Evaluation of effectiveness of marketing communication mix elements in Nigerian service sector," Pakistan Journal of Social Sciences, vol. 7, no. 2, pp. 7680, 2010.

[2] C. T. Allen, S. Fournier, and F. Miller, "Brands and their meaning makers," in Handbook of Consumer Psychology, C. P. Haugtvedt, P. M. Herr, and F. R. Kardes, Eds., pp. 781-823, Taylor \& Francis, London, UK, 2008.

[3] J. Tirole, The Theory of Industrial Organization, The MIT press, 1988.

[4] S. Krishnamurthy, "Enlarging the Pie vs. increasing one's slice: an analysis of the relationship between generic and brand advertising," Marketing Letters, vol. 11, no. 1, pp. 37-48, 2000.

[5] S. Krishnamurthy, "The effect of provision points on generic advertising funding," Marketing Letters, vol. 12, no. 4, pp. 315$325,2001$.

[6] M. P. Espinosa and P. Mariel, "A model of optimal advertising expenditures in a dynamic duopoly," Atlantic Economic Journal, vol. 29, no. 2, pp. 135-161, 2001.

[7] A. Prasad and S. P. Sethi, "Competitive advertising under uncertainty: a stochastic differential game approach," Journal of Optimization Theory and Applications, vol. 123, no. 1, pp. 163-185, 2004.

[8] F. M. Bass, A. Krishnamoorthy, A. Prasad, and S. P. Sethi, "Generic and brand advertising strategies in a dynamic duopoly," Marketing Science, vol. 24, no. 4, pp. 556-568, 2005.

[9] G. M. Erickson, "Differential game models of advertising competition," European Journal of Operational Research, vol. 83, no. 3, pp. 431-438, 1995. 
[10] M. P. Brady, "Advertising effectiveness and spillover: simulating strategic interaction using advertising," System Dynamics Review, vol. 25, no. 4, pp. 281-307, 2009.

[11] B. Viscolani, "Pure-strategy Nash equilibria in an advertising game with interference," European Journal of Operational Research, vol. 216, no. 3, pp. 605-612, 2012.

[12] G. Norman, L. Pepall, and D. Richards, "Generic product advertising, spillovers, and market concentration," American Journal of Agricultural Economics, vol. 90, no. 3, pp. 719-732, 2008.

[13] X. He, A. Prasad, and S. P. Sethi, "Cooperative advertising and pricing in a dynamic stochastic supply chain: feedback stackelberg strategies," Production and Operations Management, vol. 18, no. 1, pp. 78-94, 2009.

[14] M. M. Seyedesfahani, M. Biazaran, and M. Gharakhani, "A game theoretic approach to coordinate pricing and vertical co-op advertising in manufacturer-retailer supply chains," European Journal of Operational Research, vol. 211, no. 2, pp. 263-273, 2011.

[15] R. Ahluwalia, H. R. Unnava, and R. E. Burnkrant, "The moderating role of commitment on the spillover effect of marketing communications," Journal of Marketing Research, vol. 38, no. 4, pp. 458-470, 2001.

[16] Y. Li, L. Xu, and D. Li, "Examining relationships between return policy, product quality, and pricing strategy in online direct selling," International Journal of Production Economics, vol. 144, no. 2, pp. 451-460, 2013. 


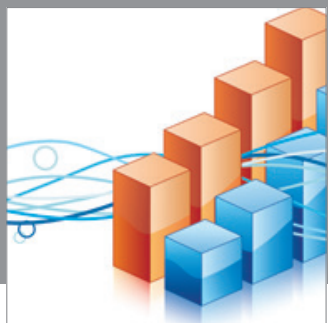

Advances in

Operations Research

mansans

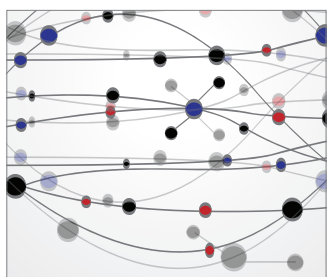

The Scientific World Journal
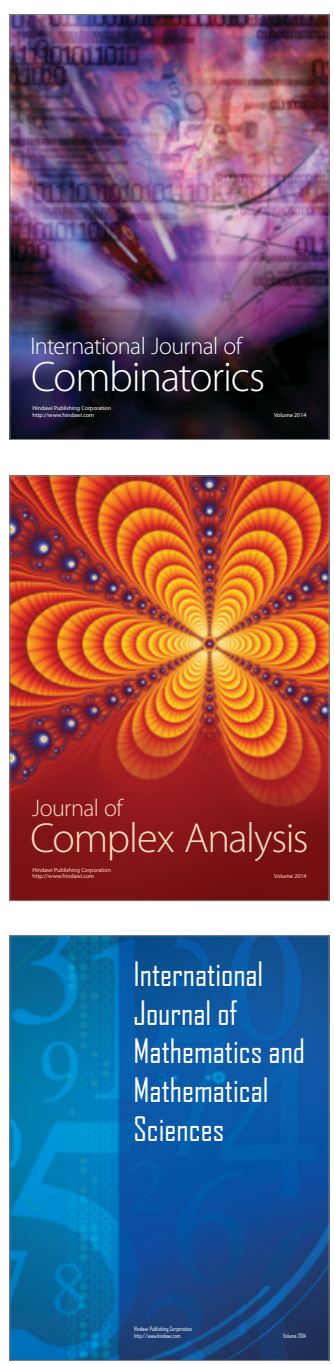
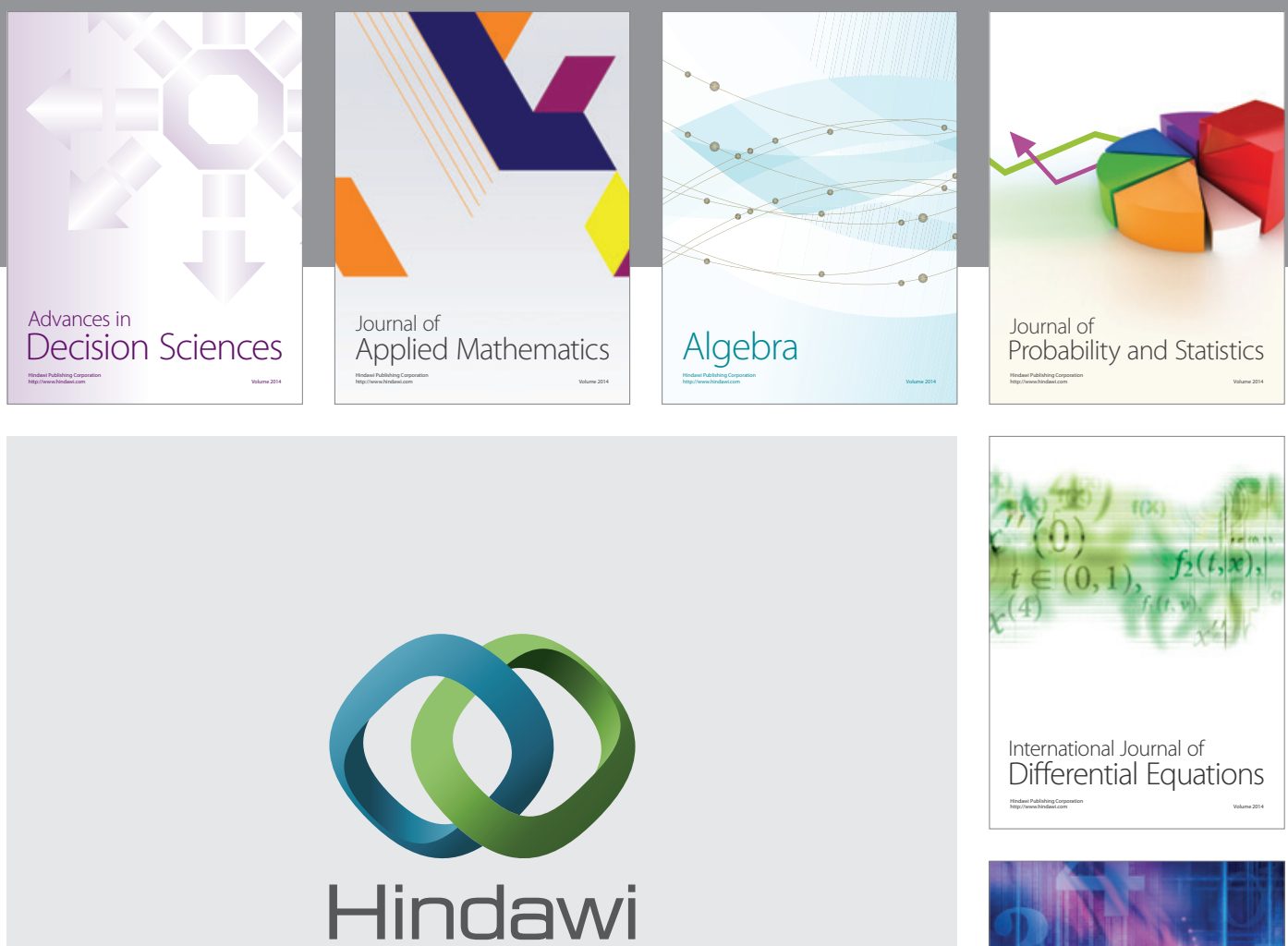

Submit your manuscripts at http://www.hindawi.com
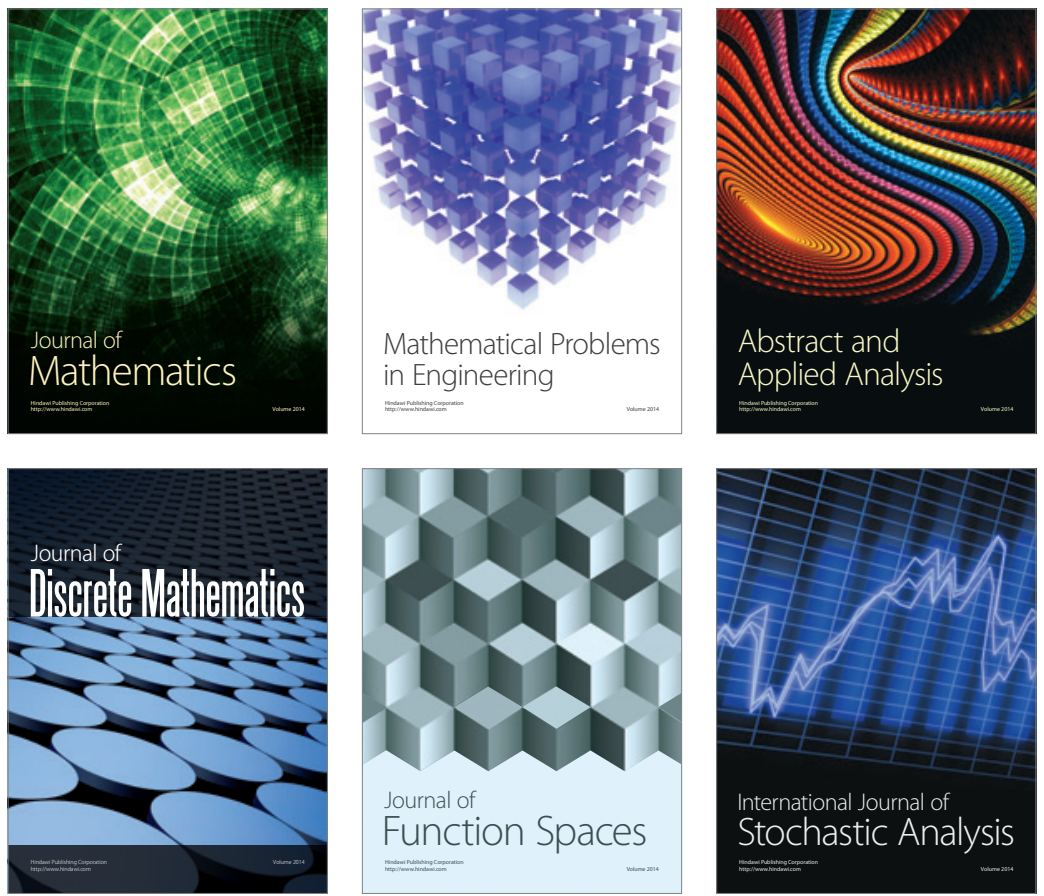

Journal of

Function Spaces

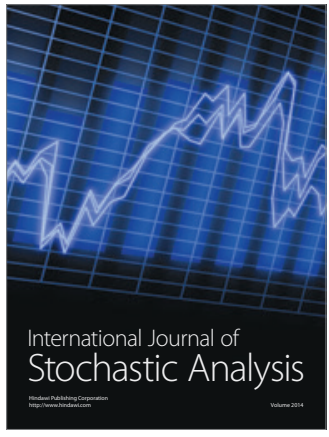

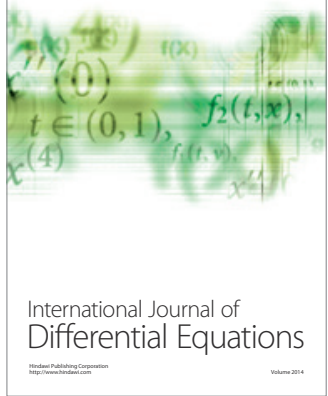
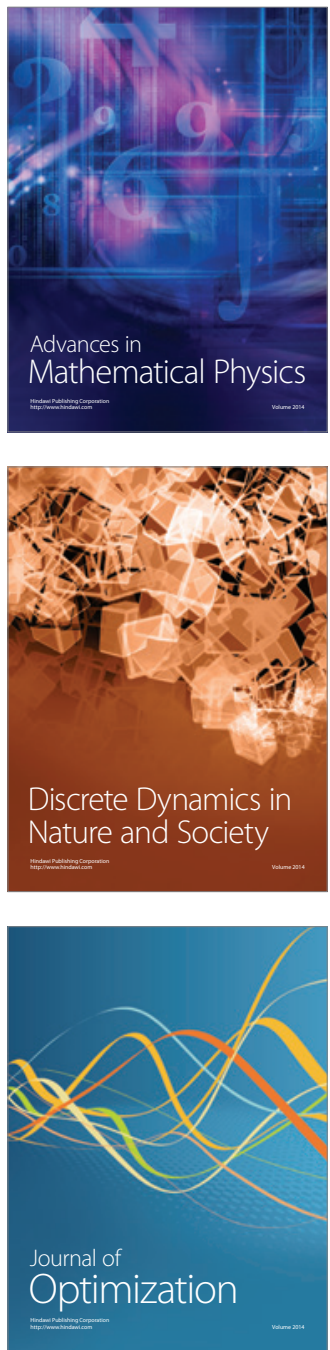